\title{
Pattern Classification Based on a Piecewise Multi-linear Model for the Class Probability Densities
}

\author{
Edgard Nyssen, Luc Van Kempen, and Hichem Sahli \\ Vrije Universiteit Brussel \\ Vakgroep Elektronica en Informatieverwerking (ETRO) \\ Pleinlaan 2, \\ B-1050 Brussel \\ BELGIUM \\ ehnyssen@etro.vub.ac.be
}

\begin{abstract}
When a Bayesian classifier is designed, a model for the class probability density functions (PDFs) has to be chosen. This choice is determined by a trade-off between robustness and low complexity - which is usually satisfied by simple parametric models, based on a restricted number of parameters - and the model's ability to fit a large class of PDFs — which usually requires a high number of model parameters. In this paper, a model is introduced, where the class PDFs are approximated as piecewise multi-linear functions (a generalisation of bilinear functions for an arbitrary dimensionality). This model is compared with classical parametric and non-parametric models, from a point of view of versatility, robustness and complexity. The results of classification and PDF estimation experiments are discussed.
\end{abstract}

\section{Introduction}

Bayesian pattern classification consists of selecting, for a given pattern $\omega$, the class $\Omega_{t}$ which maximises $\mathrm{d}_{t}(\bar{x})=\mathrm{P}\left(\omega \in \Omega_{t}\right) \mathrm{f}\left(\bar{x} \mid \omega \in \Omega_{t}\right)$, where $\bar{x}$ is the feature vector observed for the pattern. Beside the knowledge of the class prior probabilities $\mathrm{P}\left(\omega \in \Omega_{t}\right)$, the evaluation of the right hand side expression requires the class probability density functions (PDFs) $\mathrm{f}\left(\bar{x} \mid \omega \in \Omega_{t}\right)$. These class probability densities are estimated from a training set.

Examples of classical techniques are linear and quadratic discriminant analysis, which are based on modelling the functions $\mathrm{f}\left(\bar{x} \mid \omega \in \Omega_{t}\right)$ by PDFs satisfying the normal distribution (e.g. [1/23]). The parameters (the expectations of the features and their variances and covariances) are estimated from the training set data. The discriminant functions $\mathrm{d}_{t}$ obtained this way (after simplification) are linear or quadratic as suggested. For a large class of distributions, the linear and quadratic discriminant analysis techniques are quite robust to departures from the normal distribution model. For some data distributions however, these techniques don't yield satisfactory classifiers (especially, when dealing with 
multi-modal distributions). To some extent, these problems can be dealt with by considering the estimated density as a mixture of two or more probability densities of a given model - like the approach of [4], involving normal distributions. Independence from model constraints can be achieved by using nonparametric density estimation. One classical non-parametric approach is based on an approximation $\mathrm{f}_{t}$ of the probability densities using weighted sums of chosen orthonormal base functions (e.g. [2]):

$$
\mathrm{f}_{t}(\bar{x})=\sum_{j=1}^{m} \alpha_{t j} \psi_{j}(\bar{x})
$$

where $\alpha_{t j}$ are a-priori unknown coefficients. This approach searches a solution for these coefficients, minimising the criterion:

$$
C=\int_{\bar{x} \in I}\left(\mathrm{f}\left(\bar{x} \mid \omega \in \Omega_{t}\right)-\mathrm{f}_{t}(\bar{x})\right)^{2} \mathrm{~d} \bar{x} .
$$

It can be proven that the optimal coefficients may be estimated as:

$$
\hat{\alpha}_{t i}=\frac{1}{p_{t}} \sum_{\omega_{l} \in \Omega_{t}} \psi_{i}\left(\bar{x}_{l}\right),
$$

where $p_{t}$ is the number of learning set patterns of class $t$.

The advantage of the latter technique is its extreme simplicity. Practice shows however that the choice of the orthonormal base functions and the size of the expansion is not always an easy task. When $m$, the number of base functions in the expansion, is too small, a good representation of the real density function by the series expansion is not guaranteed; a high $m$-value requires however a large training set for a reliable estimation of the $m$ coefficients; moreover, the complexity of the calculation of probability density values increases with $m$. In order to tackle the latter problem, we decided to consider an approach involving an extremely simple interpolation model: the approximated probability densities are modelled as piecewise multi-linear (PML) functions. We will show that this is equivalent to decomposing the PDFs with specific localised base functions. Other approaches have been reported involving orthogonal localised base functions like wavelets (e.g.: [5]). By choosing the PML approach we opted for a model yielding a relatively fast pattern classification, at the expense of a relatively slow classifier training phase, which is due to the fact that the chosen base functions are not orthogonal.

\section{Mathematical Background of the Proposed Method}

\subsection{Problem Description}

The present paper proposes an alternative representation of approximated PDFs $\mathrm{f}_{t}(\bar{x})$, defined in a bounded domain $I$. This domain is divided into cells on the 
basis of a multidimensional rectangular point lattice. The probability densities inside the cells are obtained by a multi-linear interpolation of probability density values at the lattice points (i.e. inside a cell and along any line segment, parallel to one of the main axes of the coordinate system, values are obtained by linear interpolation). The description of a function $\mathrm{f}_{t}(\bar{x})$, is reformulated as a weighted sum of base functions, so that a reasoning can be applied, similar to the one used for the decomposition in orthonormal base functions.

\subsection{Definitions and Notations}

Before continuing the elaboration of the mathematics, we introduce here a few notations.

- considering bounded values for the $n$ components of a feature vector $\bar{x}$, its components $x_{i}(i \in\{1, \ldots, n\})$ satisfy: $x_{i \min } \leq x_{i} \leq x_{i \max }$; consequently, we define $I$ as:

$$
\bar{x} \in I=\prod_{i=1}^{n}\left[x_{i \min }, x_{i \max }\right] \subset \mathbb{R}^{n} ;
$$

- each point in the lattice is characterised by $n$ indices $r_{i}\left(r_{i} \in\left\{0, \ldots, m_{i}\right\}\right)$; to denote the indices of lattice point $\left(x_{1, r_{1}}, x_{2, r_{2}}, \ldots, x_{n, r_{n}}\right)^{\tau}$, we will use a vector $\bar{r}=\left(r_{1}, \ldots, r_{n}\right)$ as index; thus, a lattice point will be denoted by $\bar{x}_{\bar{r}}$ from now on; the set of lattice point indices $R$ is defined as:

$$
\bar{r} \in R=\prod_{i=1}^{n}\left\{0, \ldots, m_{i}\right\} \subset \mathbb{N}^{n} ;
$$

- the following subsets of lattice point indices are also defined:

$$
\begin{aligned}
& R_{\overline{0}}^{+}=\{0,1\}^{n} ; \\
& R_{\bar{r}}^{+}=\left\{\bar{r}^{\prime} \mid \bar{r}, \bar{r}^{\prime} \in R \wedge \exists \bar{r}^{\prime \prime} \in R_{\overline{0}}^{+}: \bar{r}^{\prime}=\bar{r}+\bar{r}^{\prime \prime}\right\} ; \\
& R_{\bar{r}}=\bigcup \bigcup_{\bar{r}^{\prime} \in R_{\bar{r}^{\prime}}^{-}}^{+} ; \\
& R^{-}=\left\{\bar{r}^{\prime} \mid \bar{r}^{\prime} \in R \wedge \forall i \in\{1, \ldots, n\}: r_{i}^{\prime} \neq m_{i}\right\} ;
\end{aligned}
$$

- the lattice determines $(n-1)$-dimensional hyper-planes in $\mathbb{R}^{n}$, dividing $I$ into cells $I_{\bar{r}}^{+}\left(\bar{r} \in R^{-}\right)$:

$$
I_{\bar{r}}^{+}=\left\{\bar{x} \mid \bar{x} \in I, \forall i \in\{1, \ldots, n\}: x_{i, r_{i}} \leq x_{i} \leq x_{i, r_{i}+1}\right\}
$$

\subsection{Description of the Model}

As mentioned, we introduce a piecewise multi-linear model for $\mathrm{f}_{t}(\bar{x})$ :

$$
\forall \bar{r} \in R^{-}, \forall \bar{x} \in I_{\bar{r}}^{+} \quad: \quad \mathrm{f}_{t}(\bar{x})=\sum_{\bar{r}^{\prime} \in R_{\bar{r}}^{+}} f_{t, \bar{r}^{\prime}} \prod_{i=1}^{n}\left(1-q_{i}\right)^{\delta_{r_{i} r_{i}^{\prime}}} q_{i}^{\left(1-\delta_{r_{i} r_{i}^{\prime}}\right)}
$$


where

$$
\forall i \in\{1, \ldots, n\} \quad: \quad q_{i}=\frac{x_{i}-x_{i, r_{i}}}{x_{i, r_{i}+1}-x_{i, r_{i}}}
$$

and where $\delta$ is Kronecker's symbol. The coefficients $f_{t, \bar{r}^{\prime}}$ are the PDF values at the corner points $\bar{r}^{\prime} \in R_{\bar{r}}^{+}$of cell $I_{\bar{r}}^{+}$. This is illustrated in Fig. 1 for a dimensionality of $n=2$. Note that inside cell $I_{\bar{r}}^{+}$, the function is linear in any variable $q_{i}$

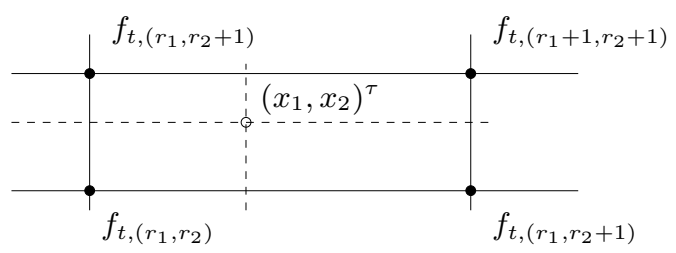

Fig. 1. Piecewise bilinear probability density function model: inside cell $I_{\left(r_{1}, r_{2}\right)}^{+}$, the values of the approximated $\mathrm{PDF}$ are found by applying bilinear interpolation: $\mathrm{f}_{t}\left(x_{1}, x_{2}\right)=f_{t,\left(r_{1}, r_{2}\right)}\left(1-q_{1}\right)\left(1-q_{2}\right)+f_{t,\left(r_{1}, r_{2}+1\right)}\left(1-q_{1}\right)\left(q_{2}\right)+f_{t,\left(r_{1}+1, r_{2}\right)}\left(q_{1}\right)\left(1-q_{2}\right)$ $+f_{t,\left(r_{1}+1, r_{2}+1\right)}\left(q_{1}\right)\left(q_{2}\right) ; q_{1}$ and $q_{2}$ satisfy (5) and take values, ranging from 0 to 1 .

for constant values of $q_{j},(\forall j \neq i)$, which means that inside a cell, the function implements a linear interpolation along the directions of the main coordinate axes.

Consider now a special set of "unit" piecewise multi-linear functions $\Psi_{\bar{r}}(\bar{x})$, defined on the basis of the same point lattice, and satisfying:

$$
\Psi_{\bar{r}}\left(\bar{x}_{\bar{r}^{\prime}}\right)=\delta_{\bar{r} \bar{r}^{\prime}}=\prod_{i=1}^{n} \delta_{r_{i} r_{i}^{\prime}},
$$

meaning that the only point in the lattice, where the value of the function is non-zero (1, actually) is the point with index $\bar{r}$. For these functions, (4) can be rewritten as: $\forall \bar{r}^{\prime \prime} \in R^{-}, \forall \bar{x} \in I_{\bar{r}^{\prime \prime}}^{+}$:

$$
\begin{aligned}
& \Psi_{\bar{r}}(\bar{x})=\sum_{\bar{r}^{\prime} \in R_{\bar{r}^{\prime \prime}}^{+}} \delta_{\bar{r} \bar{r}^{\prime}} \prod_{i=1}^{n}\left(1-q_{i}\right)^{\delta_{r_{i}^{\prime \prime} r_{i}^{\prime}}} q_{i}^{\left(1-\delta_{r_{i}^{\prime \prime} r_{i}^{\prime}}\right)} \\
& = \begin{cases}\prod_{i=1}^{n}\left(1-q_{i}\right)^{\delta_{r_{i}^{\prime \prime} r_{i}}} q_{i}^{\left(1-\delta_{r_{i}^{\prime \prime} r_{i}}\right)} \bar{r} \in R_{\bar{r}^{\prime \prime}}^{+} \\
0 & \bar{r} \notin R_{\bar{r}^{\prime \prime}}^{+}\end{cases}
\end{aligned}
$$

Functions $\Psi_{\bar{r}}(\bar{x})$ can be used as base functions for the decomposition of $\mathrm{f}_{t}(\bar{x})$ :

$$
\mathrm{f}_{t}(\bar{x})=\sum_{\bar{r} \in R} f_{t, \bar{r}} \Psi_{\bar{r}}(\bar{x}) .
$$


This allows us to express the approximation criterion of (2) as a function of unknown coefficients $f_{t, \bar{r}}$, by substituting $\mathrm{f}_{t}(\bar{x})$ by the right hand side of this equation. Equating (half) the derivatives of $C$, with respect to these coefficients, to zero, yields a system of equations: $\forall \bar{r} \in R$ :

$$
\int_{\bar{x} \in I}\left(\mathrm{f}\left(\bar{x} \mid \omega \in \Omega_{t}\right)-\sum_{\bar{r}^{\prime} \in R} f_{t, \bar{r}^{\prime}} \Psi_{\bar{r}^{\prime}}(\bar{x})\right) \Psi_{\bar{r}}(\bar{x}) \mathrm{d} \bar{x}=\frac{1}{2} \frac{\partial C}{\partial f_{t, \bar{r}}}=0 .
$$

Rearranging the terms yields: $\forall \bar{r} \in R \quad$ :

$$
\sum_{\bar{r}^{\prime} \in R} f_{t, \bar{r}^{\prime}} \int_{\bar{x} \in I} \Psi_{\bar{r}^{\prime}}(\bar{x}) \Psi_{\bar{r}}(\bar{x}) \mathrm{d} \bar{x}=\int_{\bar{x} \in I} \mathrm{f}\left(\bar{x} \mid \omega \in \Omega_{t}\right) \Psi_{\bar{r}}(\bar{x}) \mathrm{d} \bar{x} .
$$

If the functions $\Psi_{\bar{r}}$ were orthonormal, the integral in the left hand side of (17) would be zero for $\bar{r}^{\prime} \neq \bar{r}$ and 1 for $\bar{r}^{\prime}=\bar{r}$, yielding a simple expression for estimating coefficient $f_{t, \bar{r}}$, like in (3). Fortunately the base functions $\Psi_{\bar{r}}$ are only nonzero inside the cells $I_{\bar{r}^{\prime}}^{+}$, immediately surrounding the lattice point $\bar{x}_{\bar{r}}$. The only cells involved in the calculation of the integral in the left hand side of (77), are cells $I_{\bar{r}^{\prime \prime}}^{+}$, for which both $\bar{r}$ and $\bar{r}^{\prime}$ belong to the corner points; in other words: $\bar{r} \in R_{\bar{r}^{\prime \prime}}^{+}$and $\bar{r}^{\prime} \in R_{\bar{r}^{\prime \prime}}^{+}$. Note also that the right hand side of (7) is an expression for the class conditional expectation $\mathrm{E}\left\{\Psi_{\bar{r}}(\underline{\bar{x}}) \mid \omega \in \Omega_{t}\right\}$. This expression can be estimated from a training set as the mean value for the observations of class $\Omega_{t}$ of function $\Psi_{\bar{r}}$ (like the result of (3) ). On the basis of these considerations, an estimator for the unknown lattice point potentials $f_{t, \bar{r}^{\prime}}$ can be elaborated after substituting (6) in (17) and using

$$
\int_{0}^{1} q_{i}^{2} \mathrm{~d} q_{i}=\int_{0}^{1}\left(1-q_{i}\right)^{2} \mathrm{~d} q_{i}=1 / 3, \quad \int_{0}^{1} q_{i}\left(1-q_{i}\right) \mathrm{d} q_{i}=1 / 6 .
$$

One obtains: $\forall \bar{r} \in R \quad$ :

$$
\sum_{\bar{r}^{\prime} \in R_{\bar{r}}} \hat{f}_{t, \bar{r}^{\prime}} \sum_{\substack{\bar{r}^{\prime \prime} \in R^{-} \\ \bar{r}, \bar{r}^{\prime} \in R_{\bar{r}^{\prime \prime}}^{+}}} \mathrm{V}\left(I_{\bar{r}^{\prime \prime}}^{+}\right)\left(\frac{1}{2}\right)^{n}\left(\frac{1}{3}\right)^{\sum_{i=1}^{n}\left(1-\delta_{r_{i} r_{i}^{\prime}}\right)}=\frac{1}{p_{t}} \sum_{\omega_{l} \in \Omega_{t}} \Psi_{\bar{r}}\left(\bar{x}_{l}\right)
$$

where $\mathrm{V}\left(I_{\bar{r}^{\prime \prime}}^{+}\right)$is the (hyper-)volume of cell $I_{\bar{r}^{\prime \prime}}^{+}$, obtained when transforming the variables $x_{i}$ into $q_{i}$, using (5) i.e.:

$$
\mathrm{V}\left(I_{\bar{r}^{\prime \prime}}^{+}\right)=\prod_{i=0}^{n}\left(x_{i, r_{i}^{\prime \prime}+1}-x_{i, r_{i}^{\prime \prime}}\right)
$$

Equations (9) constitute a set of \#R linear equations in the \#R unknown estimated lattice point potentials $\hat{f}_{t, \bar{r}^{\prime}}$.

\subsection{Generalisation to Unbounded Feature Spaces}

In order to apply the PML method to unbounded feature vectors $\bar{y}$, their components are mapped to $x_{i}$-values, satisfying $0<x_{i}<1$. We decided to perform this 
mapping by applying first an appropriate shifting and scaling of of the feature vector components $y_{i}$ - yielding $y_{i}^{\prime}$ — and applying the inverse logit function:

$$
\left.\operatorname{logit}^{-1}: \mathbb{R} \longrightarrow\right] 0,1\left[: \operatorname{logit}^{-1} y^{\prime}=\frac{1}{1+e^{-y^{\prime}}}\right. \text {. }
$$

This function has the same form as the cumulative logistic distribution function [6]:

$$
\mathrm{F}(y ; \mu, \sigma)=\frac{1}{1+e^{-\frac{\pi(y-\mu)}{\sigma \sqrt{3}}}},
$$

where $\mu$ and $\sigma$ are resp. the expectation and the standard deviation of $y$. This means that, by putting $(\forall i \in\{1, \ldots n\})$ :

$$
x_{i}=\operatorname{logit}^{-1}\left(\frac{\pi\left(y_{i}-\mu_{i}\right)}{\sigma_{i} \sqrt{3}}\right),
$$

where $\mu_{i}$ and $\sigma_{i}$ are the expectation and the standard deviation of the feature $y_{i}$, we achieve a maximal fit of the data to the logistic distribution and, in that sense, an optimal spreading of the values of $x_{i}$-data over the interval $] 0,1[$.

\section{Experiments}

On the basis of the PML model, described in Sect. 2 we developed algorithms for the estimation of PDFs and for the design of Bayesian classifiers. These algorithms were implemented in the C programming language on a SUN SPARCstation running under the Solaris UNIX operating system. The algorithm, based on the set of linear equations, given by (9), and which calculates the lattice node PDF estimates $\hat{f}_{t, \bar{r}}$, uses simple Gauss-Seidel iteration. The time needed in the following examples to converge to a solution is in the order of magnitude of seconds.

\subsection{Comparison with PDF Estimation on the Basis of Orthonormal Expansion}

In order to study the behaviour of the algorithms, from a point of view of estimation quality, we performed a few simple numerical simulation experiments, involving one single feature $x$. In all cases, we considered problems with the same number of unknown parameters: in the case of PML modelling, the domain of $x$ was split into 10 equal intervals (= "cells" in our terminology), in the case of orthonormal expansion we used a model based on Legendre polynomials with a maximal degree of 10 . (Note that in both cases the model is characterised by 11 parameters: for the former one, 11 lattice point PDFs, for the latter one, the weight coefficients of eleven polynomials including the zero degree polynomial). In Fig. 2, we plotted the results of experiments with different kinds of probability densities (PD) which are zero outside $I$ : a PD which is uniform for all $x \in I$, a PD which is uniform in part of $I$, and zero elsewhere, (and therefore discontinuous in $I$ ), and a PD which satisfies a normal distribution, restricted to $x \in I$. Details are described in the figure's caption. 

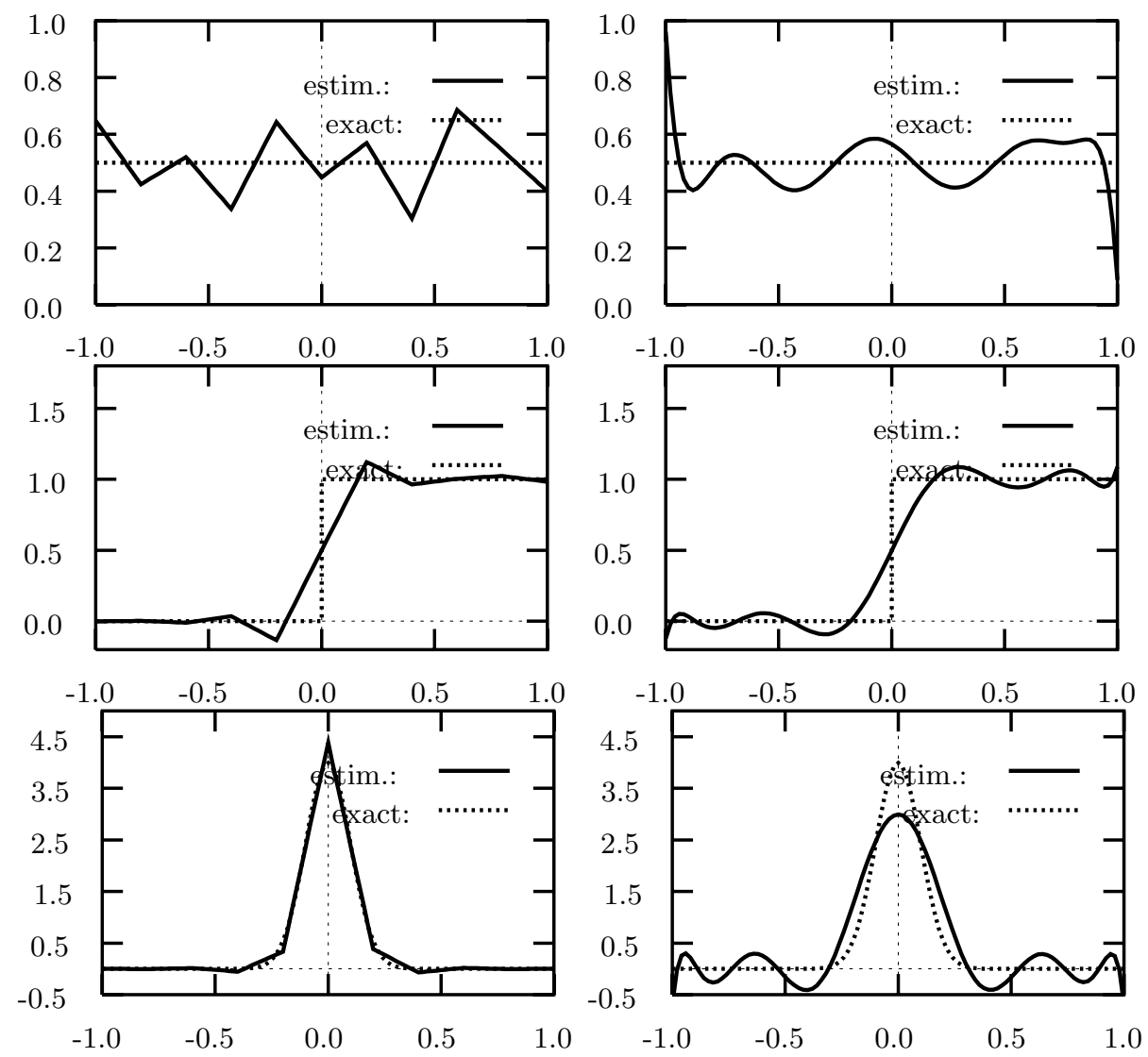

Fig. 2. Numerical univariate PDF estimation experiments; dotted lines show the exact distribution, solid lines, the estimation results: on the left side, the results based on a piecewise linear approximation, on the right side, the results based on PDF decomposition in Legendre polynomials; in the upper row, the sample size is 300 , in the middle row and lower row, the sample size is 30000; the $\sigma$ of the "clipped" normal distribution used in the lower row is 0.1 .

\subsection{Simulated Pattern Classification Experiment}

In order to verify the usefulness of the PML in circumstances where linear or quadratic discriminant analysis would fail, we performed a simulated two-class pattern classification experiment where the symmetry of the class PDFs of $\bar{x}$ causes $\bar{\mu}_{1}$ and $\bar{\mu}_{2}$ (the class expectations) and $\Sigma_{1}$ and $\Sigma_{2}$ (the class covariance matrices) to be identical. Figure 3 describes the used PDFs and the classification results in a experiment where the PML classifier involves $10^{2}$ cells.

To compare the classification results with the ones obtained on the basis of orthonormal expansion, using 14th degree Legendre polynomials of the two variables, we designed a classifier, based on this model and on the same training 


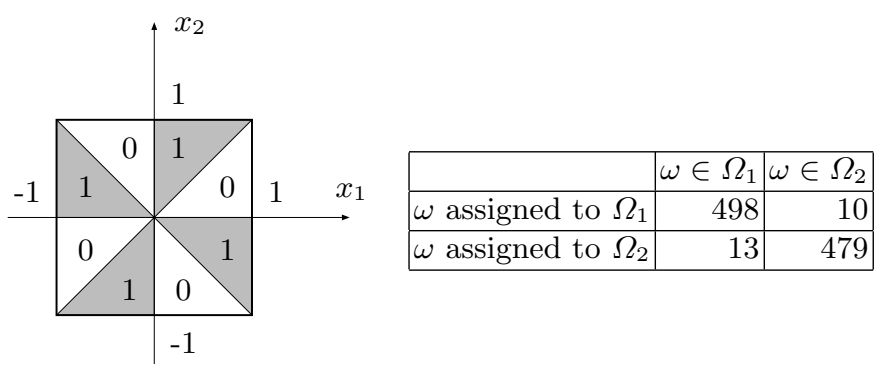

Fig. 3. Left: PDFs used in the simulated two-class pattern classification experiment: the (class independent) PDF of $\bar{x}$ is uniform inside the square; points inside the sectors, labelled 0 belong to class $\Omega_{1}$, points inside the sectors, labelled 1 belong to class $\Omega_{2}$. Right: result of classification applied to 1000 patterns of an independent test set; the model involves a lattice of 121 points; the size of the training set is 100000 .

set and applied it to the same test set; the PML function based model yields a classification efficiency of $97.7 \%$, the orthogonal expansion model, $98.4 \%$.

\subsection{Experiments on Real Measurement Data}

Finally, we performed a classification experiment, involving the Fisher iris data, used in the examples of stepwise discriminant analysis in [7. These data are well suited to demonstrate the design and application of linear and quadratic discriminant functions. We applied both discriminant analysis and PML classifier design $\left(10^{2}\right.$ cells $)$ to the two most discriminating features in the data. The training set contained 123 patterns. The classifiers were applied to 27 patterns of an independent test set; the classifier from quadratic discriminant analysis yields 3 misclassifications; the PML classifier yields 4 misclassifications. Note that the design and application of the PML classifier, involves the mapping of the data given by (10), using estimates for $\mu_{i}$ and $\sigma_{i}$.

\section{Discussion and Conclusions}

In this section, we discuss the PML approach and compare it with quadratic discriminant analysis (QDA) and orthogonal expansion (OE) - i.e. expansion in orthonormal base functions.

Versatility. The versatility of the PML model is mainly illustrated by the example of Sect. 3.2 In a situation where designing a classifier with QDA would make no sense at all, the PML model yields a high classification efficiency.

Robustness. The examples of Sect. 3.1 show that for PDFs which in theory can perfectly be matched by both the PML model and the OE model, the performances in approximating the PDF are comparable (see: Fig. 2, upper row: 
uniform distribution). However, in the presence of a discontinuity or when the approximated PDF has another kind of non-polynomial behaviour, the OE approach may yield PDF estimates showing spurious local variations throughout the whole domain, while the effect of a model mismatch has only a local effect in the PML approach (see: Fig. 2, middle and lower row). The example of Sect. 3.2 illustrates that does not necessarily imply a better classification efficiency for PML classifiers, but even in that example, the efficiencies of both the $\mathrm{OE}$ approach and PML function approach are similar. The example of Sect. 3.3 demonstrates that, compared to the classification efficiency of a classifier from QDA, the performances of the PML classifier, observed on an independent test set, are not much affected by the high number of parameters characterising this classifier (a model with $10 \times 10$ cells has $11 \times 11=121$ lattice points and an equal number of parameters $\left.f_{t, \bar{r}}\right)$.

Complexity, time consumption and memory requirements. The complexity of the algorithms in terms of elementary operations will be dependent on: the number of features $n$, the number of classes $k$, the size $p_{t}$ of the training set for each class $\Omega_{t}$ and, specifically for the PML approach, the choice for each feature $x_{i}$ of $m_{i}$ determined by the point lattice. If we limit ourselves to the operations which will be crucial for the speed of the algorithms, it is clear that, during the training phase, the PML approach is by far the most time consuming one: the calculation of the unknown lattice point potentials in (9), for one class, is an iterative process, where at each step the number of multiplications is in the order of magnitude of $3^{n} \prod_{i} m_{i}$. In both QDA and $\mathrm{OE}$, the training time is mainly determined by $p_{t}(t \in\{1, \ldots, k)$. The time spent by the PML method to calculate the right hand side of (9) is also considerable but is still small in comparison to the time consumed by the abovementioned iterative process even for moderate $n$ and relatively low $m_{i}$-values. We however did no effort until now, to optimise this stage of the training process.

The time consumption during the application of the classifier to a pattern, is much more favourable: - see: (4) and (5) - the number of multiplications is in the order of magnitude of $3 \times 2^{n}$ (which includes the calculation of the basis function values). For QDA, the number of multiplications is in the order of magnitude of $n \times(n+1)$, which is relatively small for high $n$-values. When one compares however the PML approach with $\mathrm{OE}$, one should be aware that the time consumption is independent of the total number of base functions in the PML approach (since only one cell is involved), while in $\mathrm{OE}$ - see: (11) all base functions have to be evaluated and all values have to be weighted with the corresponding coefficients $\alpha_{t j}$. When a regular lattice is used, the time to identify the involved cell and to calculate the $q_{i}$-variables can be neglected.

The memory required for the storage of the model parameters is obviously the lowest is the case of QDA, since the major part is taken by the storage of the matrices $\Sigma_{i}^{-1}$, i.e. storage for $k n^{2}$ floating point numbers. For comparable performances, the number of parameters in the OE model and the PML model are roughly equal. Like time consumption, the memory requirements are an exponential function of the number of features: in the case of a PML classifier 
model involving $\prod_{i} m_{i}$ cells for each class, a storage of $k \prod_{i}\left(m_{i}+1\right)$ floating point numbers is required. During the training of the PML model parameters this must be doubled for the storage of the right hand side of (9).

Conclusions. This paper introduces the theory and methods to estimate probability density functions on the basis of a piecewise multi-linear model. An approach has been proposed to generalise the application of the methodology to unbounded feature spaces. It has been demonstrated that the model, introduced in the present paper, is able to represent a large class of probability distributions in a satisfactory, robust way. It has also been shown that the efficiency of the derived classifiers is often better than — and at least equivalent to - the efficiency of classifiers obtained using quadratic discriminant analysis or orthogonal expansion. An attractive particularity of our approach is that almost all computation overhead is shifted to the training procedure (which only needs to be applied once for a given classifier). The classifier is fast in comparison to other classifiers of similar complexity.

\section{References}

1. Richard O. Duda and Peter E. Hart. Pattern Classification and Scene Analysis. John Wiley \& Sons, 1973.

2. Julius T. Tou and Raphael C. Gonzales. Pattern Recognition Principles. Addison Wesley Publishing Company, 1979.

3. Robert Schalkoff. Pattern Recognition - Statistical, Structural and Neural Approaches. John Wiley \& Sons, 1992.

4. Fang Sun, Shin'ichiro Omachi, and Hirotomo Aso. An algorithm for estimating mixture distributions of high dimensional vectors and its application to character recognition. In Proc. 11th Scandinavian Conference on Image Analysis, pages 267274, 1999.

5. David L. Donoho, Iain M. Johnstone, Gérard Kerkyacharian, and Dominique Picard. Density estimation by wavelet thresholding. The Annals of Statistics, 24(2):508-539, 1996.

6. N. Balakrishnan. Introduction and historical remarks. In N. Balakrishnan, editor, Handbook of the Logistic Distribution. Marcel Dekker, Inc, 1992.

7. W. J. Dixon, M. B. Brown, L. Engelman, J. W. Frane, M. A. Hill, R. I. Jennrich, and J. D. Toporek. BMDP Statistical Software 1981, 1981. 